Check for updates

Cite this: Mater. Adv., 2021,

2, 2604

Received 15th January 2021

Accepted 2nd March 2021

DOI: $10.1039 / \mathrm{d} 1 \mathrm{ma00034a}$

rsc.li/materials-advances

\section{Highly porous melamine-formaldehyde monoliths with controlled hierarchical porosity toward application as a metal scavenger $\dagger$}

\author{
Yuki Nakanishi, ${ }^{a}$ Yosuke Hara, (D) ${ }^{a}$ Riichi Miyamoto, ${ }^{a}$ Kazuki Nakanishi (D) ${ }^{\text {bc }}$ and \\ Kazuyoshi Kanamori (D)*a
}

\begin{abstract}
We report a new synthetic strategy for melamine-formaldehyde (MF) monoliths with controlled hierarchical porosity toward metal-ion scavengers. The obtained MF monoliths possessed micro-, meso- and macroporosity, which allowed efficient adsorption performance of precious metal ions in water. Applications such as recovery/removal of metal ions are expected.
\end{abstract}

Porous materials with discrete hierarchical porosity have been recognized as efficient materials for adsorption, separation, energy conversion/storage, sensing and catalysis. ${ }^{1-4}$ Various materials with micro-, meso- and macroporosity have been developed in different chemical compositions such as inorganics, organics and their hybrids in different material formats such as particles, films, and monoliths. Hierarchically porous monolithic materials are attractive among them, since they allow efficient mass transfer, which enhances the contact between the pore surface and guest fluid.,

One synthetic strategy for hierarchically porous monoliths is the sol-gel process accompanied by phase separation (spinodal decomposition). ${ }^{7}$ This strategy was originated from the silica sol-gel system, and later extended to sol-gel systems of a variety of inorganic oxides/hydroxides, organic-inorganic hybrids and organic crosslinked polymers. In the last systems, a series of hierarchically porous polymer monoliths and carbon derivatives has been prepared via controlled/living radical polymerization of

\footnotetext{
${ }^{a}$ Department of Chemistry, Graduate School of Science, Kyoto University, Kitashirakawa, Sakyo-ku, Kyoto 606-8502, Japan.

E-mail: kanamori@kuchem.kyoto-u.ac.jp

${ }^{b}$ Institute of Materials and Systems for Sustainability, Nagoya University, Furo-cho, Chikusa-ku, Nagoya, Aichi 464-8601, Japan

${ }^{c}$ Institute for Integrated Cell-Material Sciences, Kyoto University, Yoshida, Sakyo-ku, Kyoto 606-8501, Japan

$\dagger$ Electronic supplementary information (ESI) available: Detailed experimental procedure, nitrogen adsorption-desorption results of the samples without hydrothermal aging, SEM images of the hydrothermally treated samples, and results of the durability test in solvents are described in the ESI. See DOI: 10.1039/ d1ma00034a
}

divinylbenzene and other vinyl monomers/crosslinkers accompanied by phase separation. Poly(styrene-co-divinylbenzene) monoliths ${ }^{8,9}$ have been found to successfully separate small molecules such as alkylbenzenes under a low back pressure. ${ }^{10}$ On the other hand, sol-gel systems based on living polymerization often require expensive reagents and an inert atmosphere. The family of phenolic/melamine resin is another important platform toward porous materials through liquid phase processes based on the addition-condensation mechanism. These resins can be prepared with low-cost reagents under ambient conditions. Aerogels and related porous monoliths based on the phenolic resin family, first reported by Pekala ${ }^{11}$ on resorcinolformaldehyde (RF), have been attracting researchers to obtain carbonized materials with hierarchical micro- and meso- or macroporosity particularly for energy applications. ${ }^{12}$ By tuning miscibility between the phenolic resin-based network and solvent using micelle-forming surfactant, some researchers have reported hierarchically porous phenolic resin monoliths via the sol-gel process accompanied by phase separation. ${ }^{13,14}$ On the other hand, focusing on another important resin system, melamine-formaldehyde (MF), it is still challenging to control hierarchical porosity. The phase separation process in phenolic resin networks is based on the immiscibility between the rather hydrophobic phenolic resin network and hydrophilic water-based solvent. It is therefore difficult to expand the synthetic strategy for hydrophobic phenolic resin gels to the system of much less hydrophobic MF networkbased gels.

The MF network, developing through dehydrative polycondensation between methylol groups and methylol/amino groups, possesses triazine rings and amino groups in the network (Fig. $\mathrm{S} 1, \mathrm{ESI} \dagger)$. The triazine rings and amino groups are known to form complexes with metal cation species such as $\left[\mathrm{PdCl}_{4}\right]^{2-}$ through coordination and ionic interaction. ${ }^{15,16}$ Hierarchically porous MF materials are thus attractive for metal scavengers that can efficiently recover precious metal ions or remove toxic metal ions.

In the present research, we have for the first time developed a hierarchically porous monolith based on MF from a single 


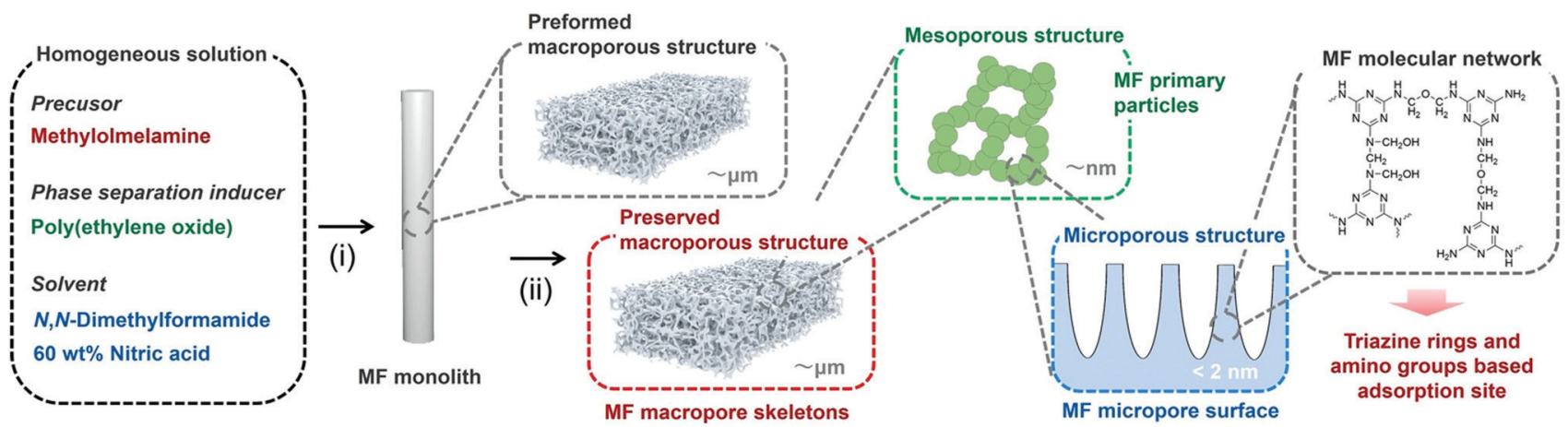

Fig. 1 Schematic of the synthesis of the hierarchically porous MF monoliths with controlled multimodal porosity. (i) Phase separation and sol-gel transition. (ii) Washing and hydrothermal treatment to form meso-/microporous structures, which imparts high specific surface area.

oligomeric precursor methylolmelamine via a simple sol-gel process, solvothermal treatment, and removing additives by a solvent exchange process (Fig. 1).

The suitable starting compositions and reaction conditions for monolithic MF gels with hierarchical porosity have been successfully specified. In a typical run, $w_{\mathrm{PEO}} \mathrm{mg}$ of poly(ethylene oxide) (PEO, $M_{\mathrm{v}} \sim 100000$ or 200000 ) was dissolved in $6.0 \mathrm{~mL}$ of $N, N$ dimethylformamide (DMF), and then $0.70 \mathrm{~g}$ of methylolmelamine was dissolved, followed by an addition of $0.10 \mathrm{~mL}$ of $60 \mathrm{wt} \%$ nitric acid. After gelation and aging at $40{ }^{\circ} \mathrm{C}$ and $60{ }^{\circ} \mathrm{C}$, the obtained monolithic gel underwent hydrothermal aging at $120{ }^{\circ} \mathrm{C}$ for $24 \mathrm{~h}$, washing by solvent exchange, and finally drying from $n$-hexane. We employed DMF, not water, as the main solvent because methylomelamine is highly soluble in DMF and a heterogeneous network tends to form in water. ${ }^{17}$ In addition, we used a small amount of $60 \mathrm{wt} \%$ nitric acid as a reaction catalyst. Under these conditions, it was allowed to form transparent gels with high enough homogeneity specifically in the absence of phase separation inducer, PEO. In the presence of PEO, polymerization-induced phase separation took place in the course of sol-gel transition due to the increased degree of polymerization of MF. The transient morphology of phase separation was then solidified by gelation of the MF network, which turned into the macroporous morphology after removing all of the fluidic components from the wet gel. Details of the experimental procedure are given in the ESI. $\dagger$

Fig. 2 shows the morphologies observed under a scanning electron microscope (SEM) in the micrometer scale of the samples obtained from the starting compositions with different molecular weight and amounts of PEO as listed in Table S1 (ESI $\dagger$ ). No distinct macropores are found in the sample MF1 (Fig. 2A), which was prepared in the presence of PEO with lower molecular weight and a smaller amount, and macroporous morphologies are found in those prepared in the presence of PEO with higher molecular weight and larger amounts (Fig. 2B-E). The samples MF3, MF4 and MF5 show a well-defined interconnected structure derived from polymerization-induced spinodal decomposition. The thermodynamic instability of this quasi twophase system composed of MF and PEO is increased with an increasing degree of polymerization (DP) of MF, and the more coarsened structures were obtained with higher DP and volume fraction of PEO, as predicted by the Flory-Huggins theory. ${ }^{18}$ These porous MF materials were obtained as colorless monoliths
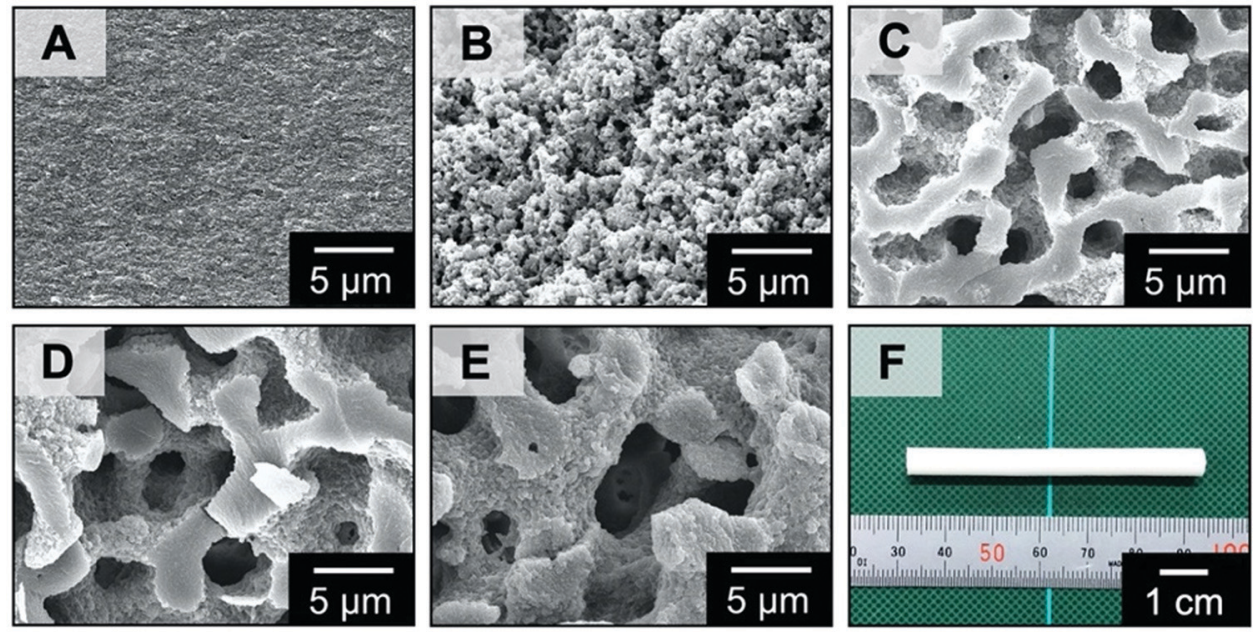

Fig. 2 (A-E) SEM images of the MF polymer monoliths: (A) MF1, (B) MF2, (C) MF3, (D) MF4, and (E) MF5. (F) Appearance of the MF polymer monoliths of MF3. 
(Fig. 2F) with high enough mechanical stability for handling and applications.

Mesoporous structures were found in the macropore skeletons of these samples, as confirmed by nitrogen adsorption-desorption isotherms and the Barrett-Joyner-Halenda (BJH) pore size distributions (Fig. S3, ESI $\dagger$ ). The higher uptake in the relative pressure $p / p^{\circ} \sim 0.8-1$ in the samples MF1 and MF2 is due to the presence of small macropores, and similar mesopore size around $4 \mathrm{~nm}$ can be confirmed in all the other samples with distinct interconnected macroporous morphology, which evidences the hierarchical porosity in these samples (see also Fig. 3 for the sample MF3). It can be deduced that most PEO was successfully removed from the wet gel during the solvent exchange process, since the attractive interaction between MF and PEO is not strong and PEO has been readily removed during solvent exchange with distilled water and 2-propanol.

In order to assess the controllability of the porous structure and extend the applicability, changes in the mesoporous structure were investigated through the hydrothermal treatment after gelation, aging and solvent exchange with distilled water on the sample MF3 (Table 1). Results of nitrogen adsorption-desorption measurements shown in Fig. 3 confirm that the mesopore diameter and total pore volume were increased with increasing temperature of hydrothermal treatment. Gels treated at $60{ }^{\circ} \mathrm{C}$ and $80{ }^{\circ} \mathrm{C}$ underwent further crosslinking of the network, resulting in the increases of pore diameter and pore volume due to decreased shrinkage during drying. At increased temperatures, dissolution and reorganization of the network became significant, which is confirmed by decreased bulk density of the dried gels, and accordingly, pore diameter and pore volume are increased drastically. The mesopore size can be controlled in $4.3-28.1 \mathrm{~nm}$ by changing the temperature of hydrothermal treatment, and the Brunauer-Emmett-Teller (BET) surface area shows maximum
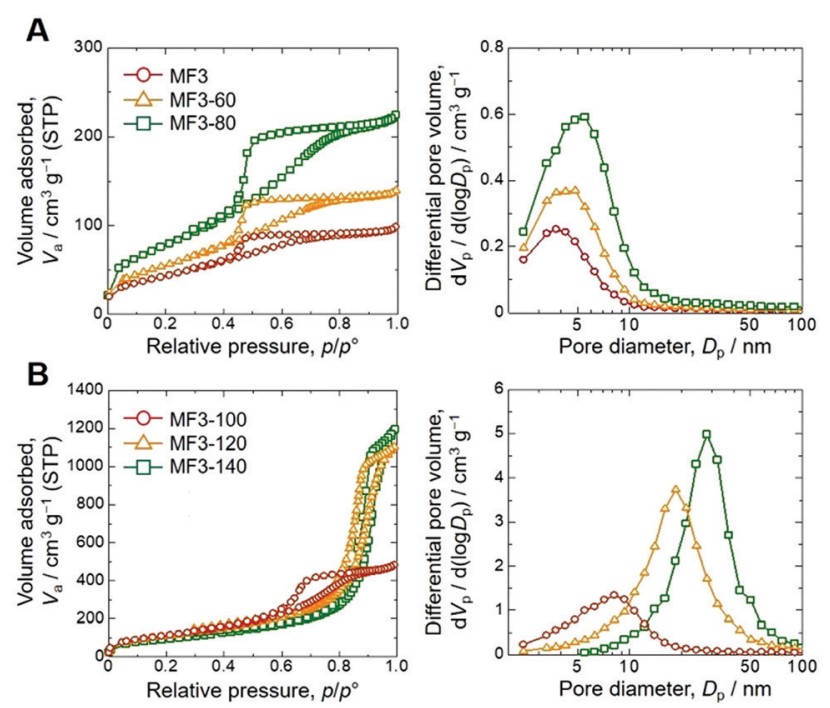

Fig. 3 Nitrogen adsorption-desorption isotherms (left) and BJH pore size distributions (right) on the MF monoliths hydrothermally treated at different temperatures: (A) as-dried MF3 and those treated at $60{ }^{\circ} \mathrm{C}$ and $80{ }^{\circ} \mathrm{C}$, and (B) those treated at $100{ }^{\circ} \mathrm{C}, 120^{\circ} \mathrm{C}$ and $140{ }^{\circ} \mathrm{C}$.
Table 1 Mesopore properties of the MF polymer monoliths hydrothermally treated at different temperatures

\begin{tabular}{llcll}
\hline Sample & $T_{\text {hydro }}{ }^{a} /{ }^{\circ} \mathrm{C}$ & $d_{\text {meso }}{ }^{b} / \mathrm{nm}$ & $V_{\mathrm{p}}{ }^{c} / \mathrm{cm}^{3} \mathrm{~g}^{-1}$ & $S_{\mathrm{BET}}{ }^{d} / \mathrm{m}^{2} \mathrm{~g}^{-1}$ \\
\hline MF3 & - & 4.3 & 0.16 & 173 \\
MF3-60 & 60 & 4.9 & 0.20 & 210 \\
MF3-80 & 80 & 5.5 & 0.33 & 296 \\
MF3-100 & 100 & 8.2 & 0.75 & 421 \\
MF3-120 & 120 & 18.5 & 1.73 & 416 \\
MF3-140 & 140 & 28.1 & 1.86 & 240
\end{tabular}

${ }^{a}$ Hydrothermal treatment temperature. ${ }^{b} \mathrm{BJH}$ modal mesopore diameter. ${ }^{c}$ Total pore volume. ${ }^{d}$ BET surface area.

(421 $\mathrm{m}^{2} \mathrm{~g}^{-1}$ ) in the sample treated at $100{ }^{\circ} \mathrm{C}$ (MF3-100). The changes in mesoporosity in the skeletons were also observed by transmission electron microscopy (TEM) as shown in Fig. 4. The original MF3 possesses micropores judging from the steep uptake in the low relative pressure region. Changes in the microporous structure in the hydrothermal treatment were estimated by the $t$-plot, ${ }^{19}$ in which the specific micropore surface area increased from $117 \mathrm{~m}^{2} \mathrm{~g}^{-1}$ in MF3 to $279 \mathrm{~m}^{2} \mathrm{~g}^{-1}$ in MF3-100. In addition, the macroporous structure did not change due to the hydrothermal treatment as confirmed by SEM as presented in Fig. S4 (ESI $\dagger$ ). The macro- and mesoporosity in the MF monoliths thus can be independently controlled through phase separation and hydrothermal treatment, respectively.

Porous MF monoliths have also been reported based on different strategies such as microemulsion templating ${ }^{20,21}$ and molecular imprinting, ${ }^{22}$ while there have been no reports on hierarchical pore formation through spinodal decomposition, to the authors' knowledge. A bimodal pore structure was obtained in the microemulsion approach; ${ }^{21}$ however, it is generally difficult to independently control the size and volume of pores in different length scales through the emulsion approach, during which random coalescence of emulsions may occur. In the present approach based on phase separation and hydrothermal aging, an independent control of macro- and mesopores with narrow size distributions is possible, since phase separation is thermodynamically driven by controlled polymerization of the monomer and mesopore formation depends on an equilibrium process of dissolution/reorganization of the network.

These MF gels showed durability in both acid and alkaline solutions, as well as organic solvents. On the treatments in $10 \%$ $\left(\sim 2.7 \mathrm{~mol} \mathrm{~L}^{-1}\right)$ hydrochloric acid, $6 \mathrm{~mol} \mathrm{~L}^{-1}$ of sodium hydroxide, and xylene at $60{ }^{\circ} \mathrm{C}$ for $24 \mathrm{~h}$, the monolithic shapes were maintained without noticeable swelling or shrinkage. The pore
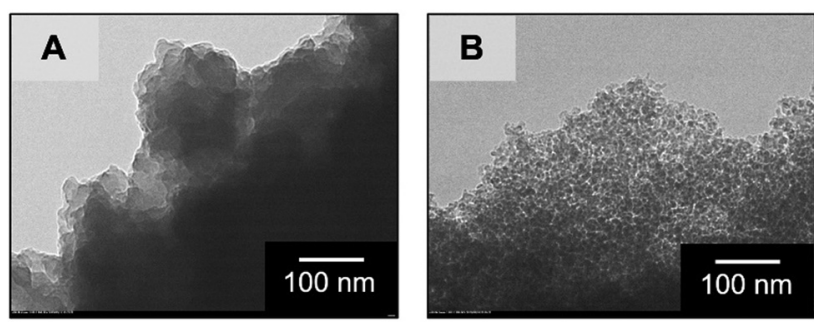

Fig. 4 TEM images of the MF polymer monoliths: (A) MF3 and (B) MF3-140. 

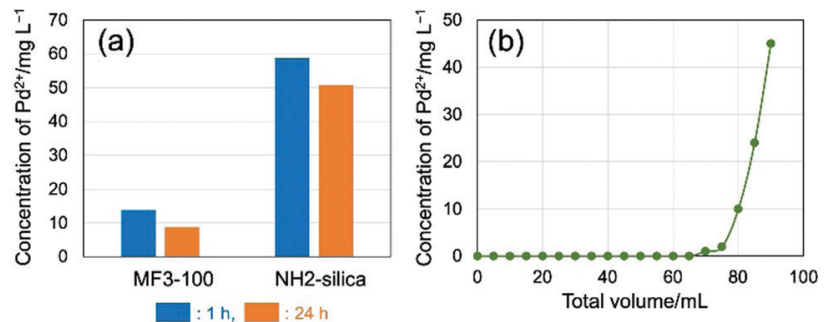

Fig. 5 (a) Concentrations of $\mathrm{Pd}^{2+}$ in the batch adsorption tests using granulated MF3-100 and aminopropyl-modified silica in $0.1 \mathrm{~mol} \mathrm{~L}{ }^{-1} \mathrm{HCl}$ with $100 \mathrm{mg} \mathrm{L}^{-1} \mathrm{Pd}^{2+}$ for 1 and $24 \mathrm{~h}$. (b) Change in the $\mathrm{Pd}^{2+}$ concentration with the total flowed volume in the flow adsorption test using granulated MF3-100

volume and mesopore diameter of the treated MF3-100 samples were slightly decreased, while the BET surface area was virtually maintained as higher than $300 \mathrm{~m}^{2} \mathrm{~g}^{-1}$ (Table S2, ESI $\dagger$ ).

Since the MF network possesses coordination sites including nitrogen in the triazine rings and amino groups, application of the present MF monoliths to metal scavengers can be expected as mentioned above. To evaluate the functionality of the MF monolith, the adsorption performance of $\mathrm{Pd}^{2+}$ species (including chloro-palladate complexes) in an acidic solution was investigated. A granulated MF monolith was used to assess the adsorption performance in a simple manner, since the benefits of high mass transfer in the macropores in the monoliths are evident in the granulated form. ${ }^{23,24}$ In particular, granulated iron oxyhydroxide and oxide monoliths with a similar macroporous morphology to the present MF material showed higher adsorption efficiency of pollutants compared to non-macroporous iron oxyhydroxide adsorbent with the same meso-/microporous structures in a flow-through setup. ${ }^{23}$ Granulated particles of dried MF3-100 in the size range between 63 and $212 \mu \mathrm{m}$ were shaken in $0.1 \mathrm{~mol} \mathrm{~L}^{-1} \mathrm{HCl}$ solution with $100 \mathrm{mg} \mathrm{L}^{-1}$ of $\mathrm{PdCl}_{2}$. Aminopropyl-modified conventional silica particles with monomodal mesopores $(\sim 6.5 \mathrm{~nm})$ and BET surface area of $\sim 450 \mathrm{~m}^{2} \mathrm{~g}^{-1}$ (denoted as NH2-silica, details shown in the ESI $\dagger$ ) were employed for comparison. The concentrations of $\mathrm{Pd}^{2+}$ after shaking for 1 and $24 \mathrm{~h}$ measured by ultraviolet-visible (UV-Vis) spectroscopy are shown in Fig. 5a. The concentrations of $\mathrm{Pd}^{2+}$ treated with MF3-100 and NH2-silica were reduced from $100 \mathrm{mg} \mathrm{L}^{-1}$ to 14 and $59 \mathrm{mg} \mathrm{L}^{-1}$ in $1 \mathrm{~h}$, and 9 and $51 \mathrm{mg} \mathrm{L}^{-1}$ in $24 \mathrm{~h}$, respectively. Most $\mathrm{Pd}^{2+}$ were adsorbed on the gel within $1 \mathrm{~h}$ effectively. The adsorbed amounts of $\mathrm{Pd}^{2+}$ on MF3-100 and NH2-silica in $24 \mathrm{~h}$ were 91 and $49 \mathrm{mg}$ g-gel ${ }^{-1}\left(0.86\right.$ and $\left.0.46 \mathrm{mmol} \mathrm{g}^{-1}\right)$, respectively. The $\mathrm{Pd}^{2+}$ concentrations were more effectively reduced in the case of MF3-100, showing higher adsorption capacity than NH2-silica, due to the abundant adsorption sites such as amino groups and triazine units in the network. Results of Fourier transform infrared spectroscopy (FTIR) on the samples MF3100 before and after adsorption of $\mathrm{Pd}^{2+}$ shown in Fig. S5 (ESI $\dagger$ ) suggest complex formations at least between nitrogen in the triazine rings and $\mathrm{Pd}^{2+}$ species. ${ }^{25}$ Since these granulated powders of MF3-100 have a discrete hierarchical pore structure consisting of macro- and mesopores in each particle, the improvement of the adsorption performance can be attributed to enhanced mass transfer and diffusion of the metal ions especially due to the macropores, compared to the conventional particles. ${ }^{26}$ The enhancement of mass transfer and diffusion and resultant improved accessibility to the pore surfaces have also been confirmed in different hierarchically porous materials. ${ }^{27-29}$

Adsorption tests have also been performed in a flow system, in which granulated powders of MF3-100 were packed in a syringe and the test solution was pressurized to flow through the bed. A typical setup for this experiment is shown in Fig. S6 (ESI $\dagger$ ). Using a small column of the granulated particles of MF3100 (packing size: $5.5 \mathrm{~mm}$ in diameter and $10 \mathrm{~mm}$ in length), the $100 \mathrm{mg} \mathrm{L}^{-1}$ of $\mathrm{Pd}^{2+}$ was reduced to less than $0.1 \mathrm{mg} \mathrm{L}^{-1}$ in a single treatment. The concentration of $\mathrm{Pd}^{2+}$ in the outlet was kept less than $0.1 \mathrm{mg} \mathrm{L}^{-1}$ during the flow treatment for at least $1 \mathrm{~h}$ at $1.0 \mathrm{~mL} \mathrm{~min}{ }^{-1}$ (Fig. $5 \mathrm{~b}$ ). The total $70 \mathrm{~mL}$ of the $\mathrm{Pd}^{2+}$ solution at $100 \mathrm{mg} \mathrm{L}^{-1}$ was able to be treated to almost completely remove $\mathrm{Pd}^{2+}$ ions using only a small column. The MF monoliths were also shown to efficiently adsorb other metal ions such as Pt, $\mathrm{Au}$ and Ir, and the adsorbed metal species can be recovered simply by incineration; hazardous liquids such as aqua regia are not necessary for extraction. These results suggest that the hierarchically porous MF monoliths can be used as an efficient precious metal scavenger.

In summary, hierarchically porous monoliths composed of the MF network have been obtained through a sol-gel process accompanied by phase separation. Mesopore properties in the macropore skeletons can be independently controlled by the hydrothermal treatment of the wet gels. The resultant MF monoliths show high stability against acid, base and organic solvent, and high adsorption efficiency for removal/recovery of $\mathrm{Pd}^{2+}$. While MF is a well-known material, still there are new opportunities in tuning the pore characteristics for more efficient applications.

\section{Conflicts of interest}

There are no conflicts to declare.

\section{Acknowledgements}

The authors thank Mr Naoki Araki and Prof. Hiroshi Kitagawa (Kyoto University) for technical support with the TEM observations. Funding: this work was financially supported by a Grantin-Aid for Scientific Research (17K06015, for K. K.) from Japan Society for the Promotion of Science.

\section{References}

1 W. Xia, A. Mahmood, R. Zou and Q. Xu, Energy Environ. Sci., 2015, 8, 1837-1866.

2 Q. Sun, Z. Dai, X. Meng and F.-S. Xiao, Chem. Soc. Rev., 2015, 44, 6018-6034.

3 X.-Y. Yang, L.-H. Chen, Y. Li, J. C. Rooke, C. Sanchez and B.-L. Su, Chem. Soc. Rev., 2017, 46, 481-558.

4 H. Sun, J. Zhu, D. Baumann, L. Peng, Y. Xu, I. Shakir, Y. Huang and X. Duan, Nat. Rev. Mater., 2019, 4, 45-60.

5 K. Nakanishi, J. Porous Mater., 1997, 4, 67-112. 
6 K. Nakanishi and N. Tanaka, Acc. Chem. Res., 2007, 40, 863-873.

7 K. Nakanishi, K. Kanamori, Y. Tokudome, G. Hasegawa and Y. Zhu, Handbook of Solid State Chemistry: 4. Nano and Hybrid Materials, ed. Andreas Stein, Wiley-VCH, Weinheim, 2017, pp. 195-241.

8 K. Kanamori, K. Nakanishi and T. Hanada, Adv. Mater., 2006, 18, 2407-2411.

9 G. Hasegawa, K. Kanamori, K. Nakanishi and T. Hanada, Carbon, 2010, 48, 1757-1766.

10 G. Hasegawa, K. Kanamori, N. Ishizuka and K. Nakanishi, ACS Appl. Mater. Interfaces, 2012, 4, 2343-2347.

11 R. W. Pekala, J. Mater. Sci., 1989, 24, 3221-3227.

12 J. Biener, M. Stadermann, M. Suss, M. A. Worsley, M. M. Biener, K. A. Rose and T. F. Baumann, Energy Emviron. Sci., 2011, 4, 656-667.

13 C. Liang and S. Dai, Chem. Mater., 2009, 21, 2115-2124.

14 G. Hasegawa, T. Shimizu, K. Kanamori, A. Maeno, H. Kaji and K. Nakanishi, Chem. Mater., 2017, 29, 2122-2134.

15 E. Birinci, M. Gülfen and A. O. Aydin, Hydrometallurgy, 2009, 95, 15-21.

16 M. X. Tan, Y. N. Sum, J. Y. Ying and Y. Zhang, Energy Environ. Sci., 2013, 6, 3254-3259.

17 Y. Nakanishi, Y. Hara, W. Sakuma, T. Saito, K. Nakanishi and K. Kanamori, ACS Appl. Nano Mater., 2020, 3, 49-54.

18 P. J. Flory, Principles of polymer chemistry, Cornell University Press, New York, 1953.
19 J. Rouquerol, F. Rouquerol and K. S. W. Sing, Adsorption by Powders and Porous Solids: principles, Methodology and Applications, Academic Press, London, 1999, pp. 222-224.

20 C. du Fresne von Hohenesche, D. F. Schmidt and V. Schädler, Chem. Mater., 2008, 20, 6124-6129.

21 C. C. Egger, C. du Fresne, D. Schmidt, J. Yang and V. Schädler, J. Sol-Gel Sci. Technol., 2008, 48, 86-94.

22 M. Liu, T. M. Tran, A. A. A. Elhaj, S. B. Torsetnes, O. N. Jensen, B. Sellergren and K. Irgum, Anal. Chem., 2017, 89, 9491-9501.

23 Y. Hara, K. Kanamori, K. Morisato, R. Miyamoto and K. Nakanishi, J. Mater. Chem. A, 2018, 6, 9041-9048.

24 W. Smits, K. Nakanishi and G. Desmet, J. Chromatogr. A, 2016, 1429, 166-174.

25 M. Maghami, F. Farzaneh, J. Simpson and A. Moazeni, Polyhedron, 2014, 73, 22-29.

26 G. Guiochon, J. Chromatogr. A, 2007, 1168, 101-168.

27 S. Dutta, A. Bhaumik and K. C.-W. Wu, Energy Environ. Sci., 2014, 7, 3574-3592.

28 M. A. Isaacs, N. Robinson, B. Barbero, L. J. Durndell, J. C. Manayil, C. M. A. Parlett, C. D'Agostino, K. Wilson and A. F. Lee, J. Mater. Chem. A, 2019, 7, 11814-11825.

29 T. Yamada, T. Matsuo, A. Ogawa, T. Ichikawa, Y. Kobayashi, H. Masuda, R. Miyamoto, H. Bai, K. Meguro, Y. Sawama, Y. Monguchi and H. Sajiki, Org. Process Res. Dev., 2019, 23, 462-469. 